\begin{tabular}{|c|l|}
\hline Title & Transformation between uninterrupted and interrupted speeds for urban road applications \\
\hline Author(s) & Pueboobpaphan, Rattaphol; Nakatsuji, Takashi; Suzuki, Hironori; Kawamura, A kira \\
\hline Citation & Transportation Research Record, 1934, 72-82 \\
\hline Issue Date & 2005 \\
\hline Doc URL & http:/hdl.handle.net/2115/8503 \\
\hline Rights & $\begin{array}{l}\text { An edited version of this paper was published in Transportation Research Record: Journal of the Transportation } \\
\text { Research Board, No. 1934, pp. 72-82, (2005). For more information, contact Transportation Research Board of the } \\
\text { National A cademies, Washington, D.C., www.TRB.org. }\end{array}$ \\
\hline Type & article (author version) \\
\hline File Information & \begin{tabular}{l} 
TRR_1934_72-82.pdf \\
\hline
\end{tabular} \\
\hline
\end{tabular}

Instructions for use 


\title{
Transformation between Uninterrupted and Interrupted Speed for Urban Road Applications
}

\author{
Rattaphol Pueboobpaphan* \\ Graduate Student \\ Transportation and Traffic Systems \\ Graduate School of Engineering, Hokkaido University \\ Kita-13, Nishi-8, Kita-ku, Sapporo 060-8628, Japan \\ Tel: +81-11-706-6822 \\ Fax: +81-11-706-6216 \\ Email: ratta@eng.hokudai.ac.jp \\ Takashi Nakatsuji \\ Associate Professor, Dr. Eng. \\ Transportation and Traffic Systems \\ Graduate School of Engineering, Hokkaido University \\ Kita-13, Nishi-8, Kita-ku, Sapporo 060-8628, Japan \\ Tel: +81-11-706-6215 \\ Fax: +81-11-706-6216 \\ Email: naka@eng.hokudai.ac.jp \\ Hironori Suzuki \\ Research Engineer \\ Safety \& ITS Research Division \\ Japan Automobile Research Institute \\ 2530 Karima, Tsukuba, Ibaraki, 305-0822 Japan \\ Tel: +81-298-56-0874 \\ Fax: +81-298-56-0874 \\ Email: hsuzuki@jari.or.jp \\ Akira Kawamura \\ Associate Professor, Dr. Eng. \\ Department of Civili Engineering \\ Kitami Institute of Technology \\ 165 Koen-cho, Kitami 090-8507 Japan \\ Tel: +81-157-26-9510 \\ Fax: +81-157-26-9510 \\ Email: kawamura@vortex.civil.kitami-it.ac.jp \\ *Corresponding Author
}

Keywords: Speed, Detector data, Probes data, Signalized intersection, Shock wave Submitted in Revised Form: November15, 2004.

Word count: 5,241 (Text) + 2,250 (1 Table +8 Figures $)=7,491$ words 


\begin{abstract}
Unlike freeway, urban traffic stream is generally interrupted by signal, and thus observed traffic data may differ from the true (uninterrupted) flow characteristic. This paper deals with the transformation between interrupted and uninterrupted speed on urban road with signalized intersection. Conventional shock wave model and two modified versions of shock wave boundary that address for the unrealistic characteristics of the conventional shock wave are developed. A method of integrating probes and detector data for speed transformation is also discussed. The proposed methods are tested with a virtual isolated signalized intersection by varying the approach demand. The numerical results suggest that all models can significantly reduce the difference between the actual and the estimated uninterrupted (or interrupted) speed compared to the case of no conversion. The applications of the proposed methods including the development of flow-speed model and link travel time estimation are also given. Limitations of the model are explained, and the areas of potential improvement are suggested.
\end{abstract}

\title{
INTRODUCTION
}

Though traffic detectors are densely deployed on urban roads in Japan, information collected by them is not fully utilized due to a lack of methodology. One of the examples is the development of traffic stream model such as flow-speed diagram from urban traffic data. This diagram provides the fundamental relationships of macroscopic traffic stream characteristics for uninterrupted flow situations (1). In case of urban roads, control devices such as traffic signal periodically interrupt traffic stream. Thus urban traffic data may not be applicable for traffic stream model development unless the uninterrupted condition is ensured. The uninterrupted condition happens only when link length is sufficiently long and the observation location is far away from the end of stopping queue at downstream signal. However, such a case is rarely seen in the center of many cities in Japan (for instance, typical link length in the center of Sapporo city is approximately $100-200 \mathrm{~m}$.). Therefore, in order to make use of urban traffic data for traffic stream model development, a method for transforming between the interrupted and uninterrupted speed is required.

Several studies have used detector data for various applications including link travel time and journey speed estimation (e.g., 2-4). The motivation of this work is to support the implementation of advanced traveler information systems (ATIS) or the better management of traffic signals. Unlike freeway, link travel time on urban road can be viewed as comprising of cruise time and signal delay, where the cruise time is the average travel time for a vehicle to traverse the link (4). In this view, the cruise time is exactly the same as the time required to traverse a link at the uninterrupted speed (or cruise speed). Hence, the ability to estimate the uninterrupted speed from interrupted speed on urban road would benefit to the estimation of link travel time.

As a result, the main objective of this paper is to investigate how to transform between the interrupted and uninterrupted speed with the focus on the application to urban road with signalized intersection. As a first stage, only the case of under-saturated condition is considered in this paper. Shock wave theory, first developed by Lighthill and Whitham (5), and Richards (6), is employed for this purpose. Among the applications, it has been used for the analysis at signalized intersection (7). The excellent feature of this method is that it concerns with queue dynamics and the effects of signal control. The first section of this paper reviews the shock wave concept for application to signalized intersection. The difference between observed speeds from different detector locations is clarified. This is followed by the illustration of how conventional shock wave analysis is used for speed conversion in case of using only detector data. The second section discusses the issues regarding the unrealistic behavior of shock wave model and proposes the modified shock wave boundaries in order to address this problem. The paper continues by developing a methodology that integrates both probes and detector data. Then numerical analyses based on simulation results are shown for all the proposed models. The example applications are also given. Finally, the conclusion is drawn and the direction for future research is discussed.

\section{SPEED TRANSFORMATION BY SHOCK WAVE THEORY}

When traffic state changes from one to another state, a boundary between these two states is established. This boundary is referred to as shock wave. A concept of shock wave at signalized intersection can be depicted in Figure 1 . The analysis can be performed if a flow-density relationship of the approach to the signalized intersection is known in advance. The speed of shock wave is calculated by 


$$
w_{i j}=\frac{q_{i}-q_{j}}{k_{i}-k_{j}}
$$

where $w_{i j}$ represents the speed of shock wave between traffic state $i$ and $j$. Symbols $q_{i}$ and $q_{j}, k_{i}$ and $k_{j}$, as well as $v_{i}$ and $v_{j}$, represent flow rate, density, and speed of any traffic state $i$ and $j$, respectively.

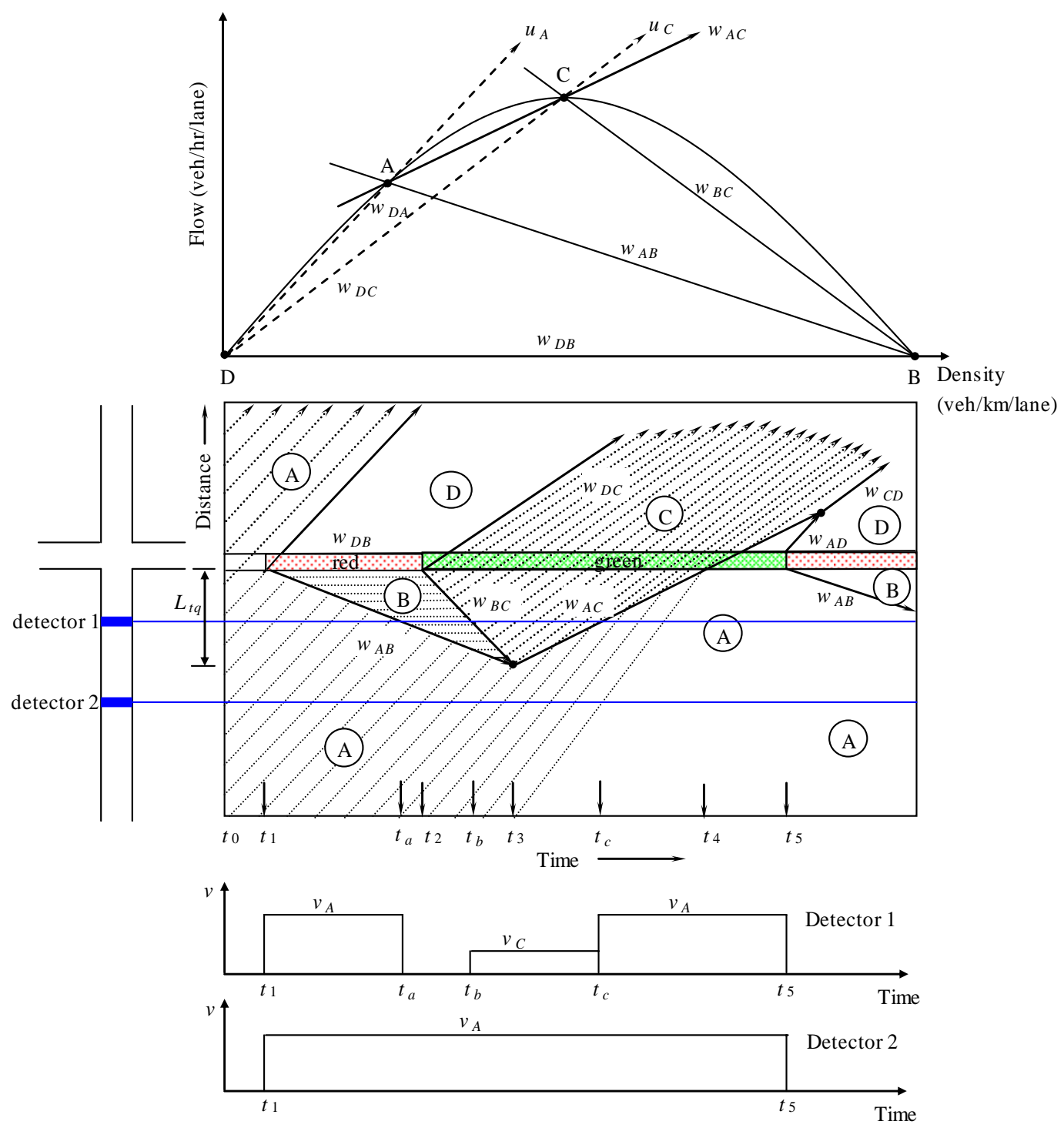

FIGURE 1 Shock Wave Analysis at Signalized Intersection (Modified from May, A. D. (1)).

According to Figure 1, traffic states $A, B, C$, and $D$ refer to the approaching traffic $\left(q_{A}\right)$, stopped traffic, saturation flow traffic $\left(q_{C}\right)$, and the zero flow traffic states, respectively. At any signal cycle, it starts from red at time $t_{1}$ and ends at time $t_{5}$. A length where the queue dissipates is defined as $L_{t q}$ and can be calculated as follows

$$
L_{t q}=\frac{w_{A B} \cdot w_{B C}}{w_{B C}-w_{A B}} \cdot r
$$

where $r$ denotes the effective red interval of the downstream signal (C-g: $C$ is cycle length, $g$ is effective green interval). From this figure, if detector location is at a distance $L_{d}$ in between the length of $L_{t q}$ from intersection (e.g., detector 1 in Figure 1), there should be some influences of signal delay to the observed speed. Average 
speed observed from detector within this area can be considered as the weighted average by time of speed in region $A$, region $B$, and region $C$. For a case that detector location is far beyond $L_{t q}$ in upstream (e.g., detector 2 in Figure 1), the observed speed would be the same as speed in region $A\left(v_{A}\right)$. According to this, it can be summarized that the observed speed is different from the uninterrupted speed only when detector location is in between the signal and distance $L_{t q}$ otherwise the observed speed is the same as $v_{A}$. The same fundamental idea is used throughout of this paper.

From Figure 1, the time that shock wave $A B$ meets shock wave $B C$ at distance $L_{t q}$ from signal is defined as $t_{3}$, and can be calculated as follows

$$
t_{3}=t_{2}+\frac{w_{A B} \cdot r}{w_{B C}-w_{A B}}
$$

where $t_{2}$ is the time that signal turns from red to green $\left(t_{1}+r\right)$. Time $t_{4}$ is the time shock wave AC crosses stop line. Shockwave $A B, B C$, and $A C$ crosses detector 1 at time $t_{a}, t_{b}$, and $t_{c}$, and can be calculated as shown in equation (4), (5), and (6), respectively.

$$
\begin{aligned}
& t_{a}=t_{1}+\frac{L_{d}}{w_{A B}} \\
& t_{b}=t_{2}+\frac{L_{d}}{w_{B C}} \\
& t_{c}=t_{3}+\frac{L_{t q}-L_{d}}{w_{A C}}
\end{aligned}
$$

The value of speed during the time between $t_{1}$ and $t_{a}, t_{a}$ and $t_{b}, t_{b}$ and $t_{c}$, and $t_{c}$ and $t_{5}$, is easily seen from Figure 1 as $v_{A}$, zero speed, $v_{C}$, and $v_{A}$, respectively. The average observed speed $\left(v_{O}\right)$ at any detector location can be summarized as follows

$$
\begin{aligned}
& v_{O}=\frac{v_{A} \cdot\left(t_{a}-t_{1}\right)+0 \cdot\left(t_{b}-t_{a}\right)+v_{C} \cdot\left(t_{c}-t_{b}\right)+v_{A} \cdot\left(t_{5}-t_{c}\right)}{C}, \text { if } L_{d} \leq L_{t q} \\
& v_{O}=v_{A} \text {, if } L_{d}>L_{t q} .
\end{aligned}
$$

The above equation requires that arrival flow rate, $q_{A}$, must be known in advance. In contrast to speed, as generally known to traffic engineers, the average observed flow rate is in fact the same as arrival flow rate as long as this arrival flow is less than or equal to capacity of intersection $c$ (saturation flow multiplied with green time ratio, $q_{C} \cdot(g / C)$ ) or in other words, the demand to capacity ratio, $q_{A} / c$, is less than or equal to 1.0 . It is also known that the average observed flow rate is equal to the capacity of intersection when $q_{A} / c>1.0$. As the starting point of this research, the analysis in this paper is limited to the case of $q_{A} / c \leq 1.0$ and thus arrival flow is equal to the observed flow from detector. The other variables that are necessary for the calculation include signal settings (C, $g$, and $r$ ); saturation flow rate, $q_{C}$; density at the saturation flow state, $k_{C}$; and the jam density, $k_{B}$. These variables are the basic variables in general traffic analysis and are assumed known in advance.

Equation (7) can be considered as the general relationship between $v_{O}$ and $v_{A}$ or in other words, the relationship between interrupted speed (include effect from signal) and the uninterrupted speed (no effect from signal). It can be used to estimate the value of $v_{O}$ if $v_{A}$ is given, or inversely estimate $v_{A}$ if $v_{O}$ is known (transformation between interrupted and uninterrupted speed).

The algorithm to transform $v_{A}$ to $v_{O}$ is straightforward. The value of $v_{A}$ is used for calculating $k_{A}$ using the basic relationship of $q=v k$. The detector location from downstream signal, $L_{d}$, is compared with $L_{t q}$ and followed by the calculation as shown in equation (7). However, the transformation from $v_{O}$ to $v_{A}$ is not easy as above since $L_{t q}$ is a function of $w_{A B}$ which is a function of $v_{A}$ itself. The procedure to solve this problem therefore relies on the trial and error basis. The calculation is performed by randomly changing the value of $v_{A}$ within the feasible region and record the value of $v_{A}$ that gives the minimum difference between $v_{O}$ estimated from equation (7) and actually observed from detector. 


\section{MODIFIED SHOCK WAVE FOR SPEED TRANSFORMATION}

The application of shock wave theory to the analysis of signalized intersection in the above section is based on a number of simplifying assumptions and limitations (1). For example, it assumes that drivers do not anticipate changes in downstream flow conditions and are assumed to change their speeds instantaneously only at shock wave boundaries. According to this, two modified forms of shock wave boundary are examined in this paper. Both are dealing with the fact that drivers need a distance for deceleration by changing the vertical boundary of shock wave $A B$ to linear and parabolic form. Later on, in this paper, these modified shock waves are referred to as "linear shock wave" and "parabolic shock wave", and the original shape of shock wave is called "original shock wave". It should be noted that only the modification of the boundary of shock wave $A B$ is performed in this paper. The modified forms and the original form are shown together in Figure 2. Figure 2(a) presents a relationship between speed and distance upstream of signal at different times. The time variables in this figure (i.e., $t_{1}, t_{2}, t_{3}, t_{4}, t_{5}$ ) are the same as in Figure 1 . The situation at time $t=t_{3}$, which is the time when queue dissipate is shown in Figure 2(b). A length $L_{1}$ in this figure is defined as a distance required by vehicles for decelerating from the speed of $v_{A}$ to stop speed, and can be calculated as follows

$$
L_{1}=\frac{v_{A}^{2}}{254(F \pm G)}
$$

where $F$ denotes the coefficient of friction between tires and roadway, $G$ represents grade of road surface in decimal ( $v_{A}$ in $\mathrm{kph}$ and $L_{1}$ in $\left.\mathrm{m}\right)$. The value of $F$ depends greatly on the road surface condition. $L_{2}$ is introduced in Figure 2 in order to merge the boundary of shock wave $A B$ (linear or parabolic) with the vertical boundary of shock wave $B C$ at time $t_{3}$. In case of linear, a same slope as used for $L_{1}$ is applied to find $L_{2}$. For parabolic shape, a same focal length as of the parabolic shape associated with length $L_{1}$ is used to define $L_{2}$. According to this definition, $L_{2}$ can be calculated as follows.

$$
\begin{aligned}
& L_{2}=\left(\frac{v_{A}-v_{C}}{v_{A}}\right) \cdot L_{1} \text {, for linear shock wave } \\
& L_{2}=\left(\frac{v_{A}-v_{C}}{v_{A}}\right)^{1 / 2} \cdot L_{1} \text {, for parabolic shock wave }
\end{aligned}
$$

From Figure 2, the linear and parabolic boundaries connect between two speed values $\left(v_{A}\right.$ and zero, $v_{A}$ and $v_{C}$ ) with length $L_{1}$ (or $L_{2}$ ). Once the length $L_{1}$ (or $L_{2}$ ) and the difference between these two speeds (i.e., $v_{A}-0$ or $v_{A}-v_{C}$ ) are calculated, the mathematical equation that describes the linear and parabolic boundaries can be defined. Then the relationship between speed and distance at any instant time can be developed. The longest distance that the right end of linear and parabolic boundaries can move far from stop line is $L_{t q}+L_{1}$ at time $t_{3-}$. If the detector location is in between stop line and a distance of $\left(L_{t q}+L_{1}\right)$, there should be some influences of signal delay to the average observed speed, and thus $v_{O}$ is different from $v_{A}$. The algorithm to convert $v_{A}$ to $v_{O}$, or vice versa, is similar in concept as those applied in the case of original shock wave. It is still relied on the weighted average of speed associated with its observed period. At this point, it is worth to note that the calculation concept is the same as in original shock wave, but the observed speeds at different point in time are not same for different shape of shock wave boundary.

\section{COMBINATION OF PROBE AND DETECTOR DATA}

For the past decade or so, traffic surveillance technologies have been improved at a rapid progress. Using probes for data collection seems to be a promising way since data obtained from probe reflects both temporal and spatial variations. In this section, a method of speed conversion from $v_{O}$, which is defined in this section as the observed speed from probe data, to $v_{A}$ is discussed. The modified form of Webster delay formula is employed for this purpose. Note that delay formula requires the arrival demand $\left(q_{A}\right)$ as the input for the calculation. Since probe data does not provide any information of flow rate, the integration of detector data for this purpose is therefore meaningful. However, the Webster formula is applicable only for $q_{A} / c<1.0$, therefore the analysis in this part is limited at $q_{A} / c<1.0$. The modified Webster formula is shown as follows 


$$
D L=0.90\left\{\frac{C}{2} \frac{[1-(g / C)]^{2}}{\left[1-\left(q_{A} / q_{C}\right)\right]}+\frac{\left(q_{A} / c\right)^{2}}{2 q_{A}\left[1-\left(q_{A} / c\right)\right]}\right\}
$$

where $D L$ is the average delay. After calculating average delay using equation (10), the relationship between $v_{A}$ and $v_{O}$ can be defined by

$$
v_{A}=\frac{L}{\left(\frac{L}{v_{O}}\right)-D L}
$$

where $L$ is link length. From this equation, the transformation from $v_{O}$ to $v_{A}$ can be performed using the same concept as in previous.
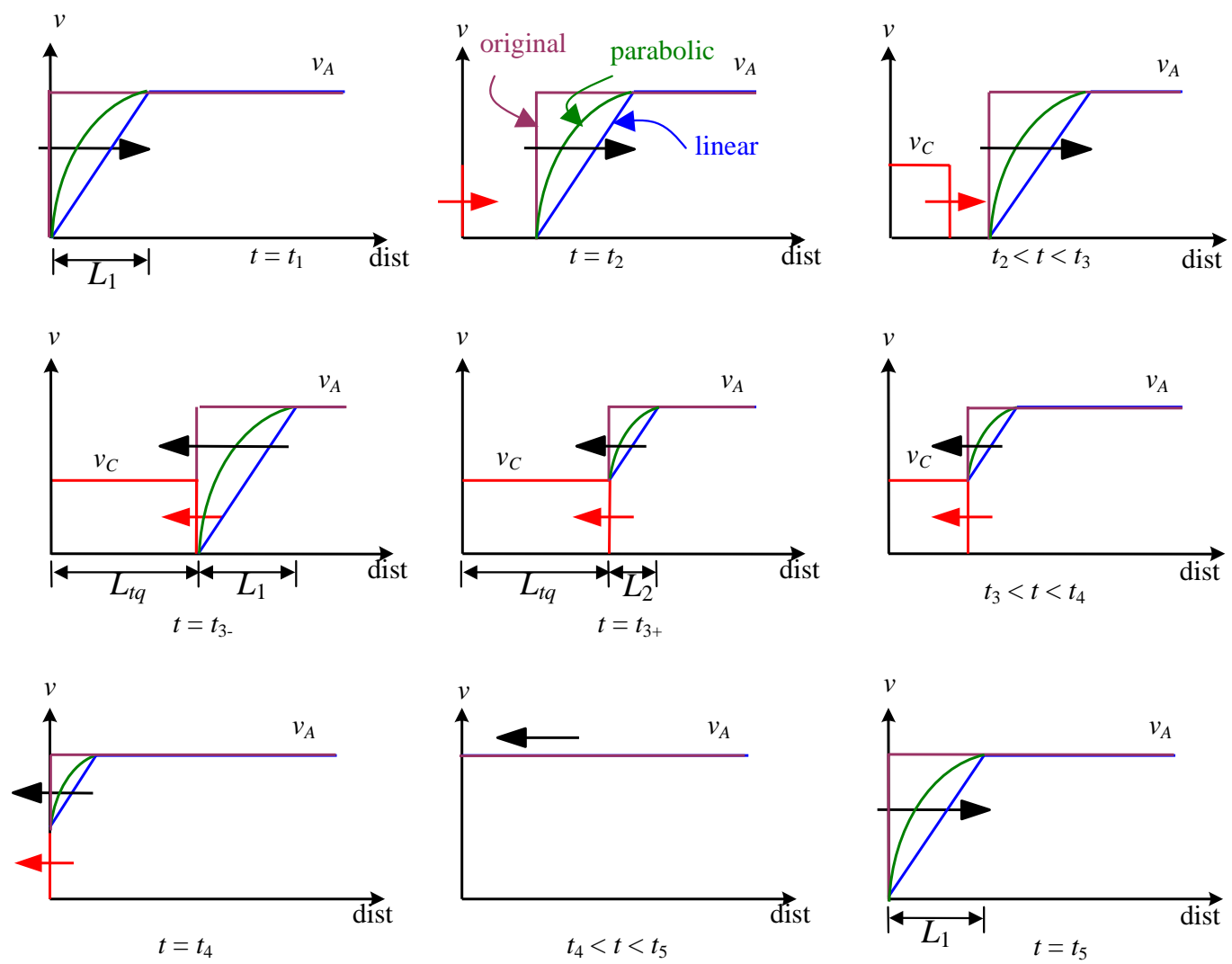

(a) Distance-Speed Diagram

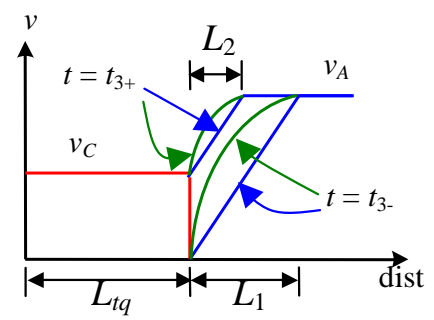

(b) Speed at Time $t_{3}$

FIGURE 2 Shock Wave and Speed Distribution Pattern. 


\section{NUMERICAL EXPERIMENT}

Due to the difficulty to find a place where many detectors were installed on a same link and also the difficulty to measure the actual value of uninterrupted speed in real world, the numerical example in this paper was based solely upon the application of simulation package, INTEGRATION (8). A virtual isolated signalized intersection was considered in this study with the approach link of one lane and one kilometer in length. The INTEGRATION requires the pre-specified value of a number of variables in order to run the model. Signal cycle length was set to 75 seconds with typical two phases. The ratio of effective green time to cycle length of the link under observation was set to 0.5 . Free flow speed was specified as $70 \mathrm{kph}$ while the speed at saturation flow, $v_{C}$, (later on it is called saturation speed) was set to $33.33 \mathrm{kph}$. A constant value of $120 \mathrm{veh} / \mathrm{km} / \mathrm{lane}$ was used for jam density, $k_{B}$. The saturation flow rate, $q_{C}$, was set to $2000 \mathrm{vph}$ while the demand to the intersection capacity ratio, $q_{A} / c$, was considered as $0.2,0.4,0.6,0.8$, and 1.0 . A total number of 50 detectors were placed on the link under study at a spacing of $10 \mathrm{~m}$ between 10 and $500 \mathrm{~m}$ from stop line. The simulation period was 4200 seconds, but only the traffic data between 300 and 3900 seconds was used in order to avoid the unstable condition during the beginning and the end of simulation period. The percentage of probes assigned in this study was 5 percent.

The outputs from INTEGRATION that were used in the analysis include the observed speed, $v_{O}$, and flow, $q_{A}$, from detector, and the travel time and speed traversing the link from probes. The actual value of $v_{A}$ that was used for the assessment of the model, however, was not directly available. In this case, a same set of input of traffic variables were used to simulate traffic data again under the same road condition, except only that the signal was taken out from the road. This results in the observed speed from detector and probes that reflecting only the interaction among vehicles. This speed was referred to the actual $v_{A}$ and used for the analysis and assessment of model.

\section{Simulation Outputs}

The average flow and time mean speed observed by detector during the simulation time between 300 and 3,900 seconds were calculated at each of detector point. The average observed value of $v_{O}, q_{A}$, and the actual value of $v_{A}$ are shown in Figure 3(a), 3(b), and 3(c), respectively. From Figure 3(a), the observed speeds at any detector location decrease as $q_{A} / c$ increase. It is clearly seen that at low volume, the observed speed tends to increase as the detector location is far away from signal. However, as the volume level changes from low to high, the observed speed tends to decrease at the short distance near to signal and increase at distance far away $\left(q_{A} / c=0.8\right.$ and 1.0). The reason for this is expected to be as follows. At very high flow, there is a high probability that some vehicles stopping on a queue at red cannot complete traversing that link within the next green time. Once signal turns to green, these vehicles start moving from stop to a quite low speed and then have to decelerate their speed due to signal turns to red again. Therefore, if one observes speed at a distance within a queue length, there is a probability that the speed at this point is lower than the speed at stop line, particularly for very high flow rate. The actual uninterrupted speed was almost flat regardless of where the detector location is. This is also same for the observed flow rate. As long as the demand is less than capacity of intersection, the average observed flow rate tends to be equal to demand. The observed speed and the actual uninterrupted speed in Figure 3(a) and 3(c) were used as the target for the results from models.

\section{Capability of the Proposed Models}

In this subsection, the comparisons between the actually observed speed and those estimated speeds from the proposed models are performed. The comparisons are separated into three parts. The first two parts deal with the use of detector data for the conversion of speed from $v_{A}$ to $v_{O}$, and the inverse conversion of speed from $v_{O}$ to $v_{A}$. The last part uses probe data in combination with detector data for converting speed from $v_{O}$ to $v_{A}$. The performance index for the comparisons between the actual and the estimated value of speed relies on the Root Mean Square Error (RMSE). The additional part on the possible applications of the proposed method is also illustrated. The results are shown in Table 1 and the discussions are provided separately for each part.

\section{Using Detector Data for Direct Conversion from Uninterrupted Speed to Observed Speed}

In this part, the conversion of uninterrupted speed is performed so that the converted speed would behave like the one actually observed from detector. Note that the value of $F$ for calculating $L_{1}$ is not available so it was assigned 


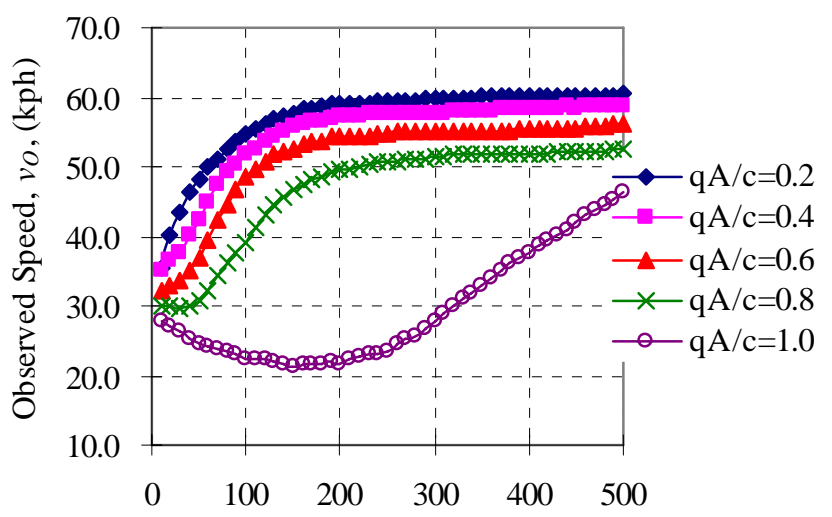

Detector Location from Downstream Signal (m)

(a) Actual Observed Speed

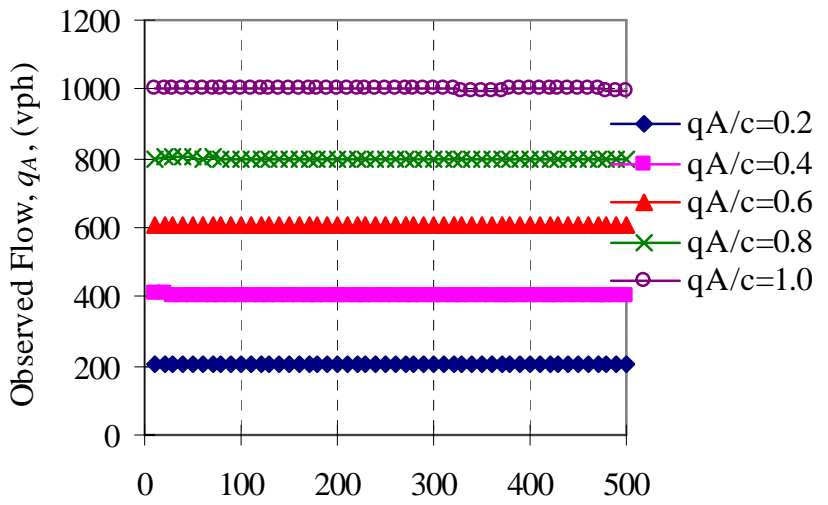

Detector Location from Downstream Signal (m)

(b) Observed Flow

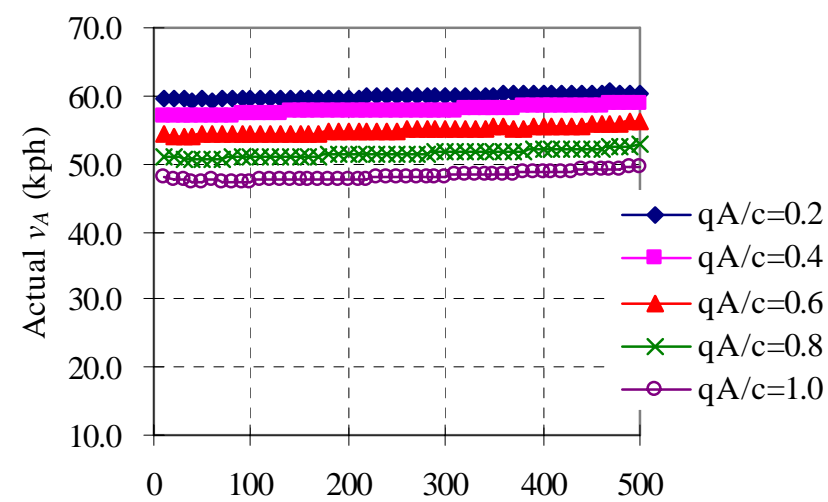

Detector Location from Downstream End (m)

(c) Actual Uninterrupted Speed

FIGURE 3 Simulation Outputs. 
between 0.1 and 0.8 , and only the most proper one was used in comparison with other models. Results from three proposed models and the actual value of $v_{O}$ are shown in Figure 4 for $q_{A} / c \leq 1.0$. Only the results from detector located within $200 \mathrm{~m}$ from signal were considered in the comparison.

TABLE 1 Root Mean Square Error (RMSE) of the Model Results

\begin{tabular}{|c|c|c|c|c|c|c|c|c|c|c|c|}
\hline \multirow{3}{*}{$q_{A} / c$} & \multirow{3}{*}{$\begin{array}{r}\text { No } \\
\text { Adjust }\end{array}$} & \multirow{2}{*}{\multicolumn{3}{|c|}{ Conversion from $v_{A}$ to $v_{O}$}} & \multicolumn{6}{|c|}{ Inverse Conversion from $v_{O}$ to $v_{A}$} & \multirow{3}{*}{$\begin{array}{r}v_{A} \text { by } \\
\text { Probe \& } \\
\text { Detector }^{4}\end{array}$} \\
\hline & & & & & \multicolumn{3}{|c|}{ No Constraint } & \multicolumn{3}{|c|}{ With Constraints } & \\
\hline & & Origin $^{1}$ & Linear $^{2}$ & Parab $^{3}$ & Origin $^{1}$ & Linear $^{2}$ & Parab $^{3}$ & Origin $^{1}$ & Linear $^{2}$ & Parab $^{3}$ & \\
\hline 0.2 & 9.51 & 8.13 & 4.11 & 3.90 & 8.32 & 7.23 & 6.79 & 8.32 & 6.42 & 5.93 & 3.72 \\
\hline 0.4 & 10.46 & 7.09 & 4.56 & 4.56 & 7.32 & 8.42 & 7.89 & 7.32 & 7.25 & 6.95 & 1.89 \\
\hline 0.6 & 11.49 & 5.54 & 4.33 & 4.02 & 6.38 & 9.71 & 8.42 & 6.37 & 6.87 & 6.64 & 0.99 \\
\hline 0.8 & 13.36 & 5.87 & 4.89 & 4.87 & 9.11 & 20.41 & 18.20 & 7.80 & 8.40 & 8.27 & 0.91 \\
\hline 1.0 & 24.33 & 17.78 & 12.94 & 14.59 & 32.66 & 17.62 & 19.93 & 14.32 & 11.86 & 12.42 & $\mathrm{NA}^{*}$ \\
\hline
\end{tabular}

Origin $^{1}$ represents the case of original shock wave model

Linear ${ }^{2}$ represents the case of linear shock wave model

Parab $^{3}$ represents the case of parabolic shock wave model

${ }^{4} v_{A}$ was calculated using equation (10) and (11)

$\mathrm{NA}^{*}$ : not applicable since the modified Webster formula is applicable only for $q_{A} / c<1.0$

It can be seen that the original shock wave model generally overestimates $v_{O}$ in almost cases. Estimation results from linear and parabolic shock wave model are quite close to each other and are more accurately than the original model from both visual assessment and the RMSE point of view. It is also found that both linear and parabolic model underestimate $v_{O}$ when detector is located near to signal and overestimate when detector is located beyond $75 \mathrm{~m}$ (approximately) from signal. For this type of estimation, the parabolic shock wave model, compared to the other two models, provides the best fit with observed speed. For $q_{A} / c=1.0$, none of model can fit well with the observed speed.

It can be seen from Figure 4 that the estimation results when the detector location is near to signal were not well enough. This might be resulted from the underlying features of shock wave. Shock wave assumes all stopped vehicles are discharged from intersection at a constant speed $v_{C}$. In reality, this may not be the case since the road ahead the intersection is free from traffic so vehicles tend to increase their speed from zero to as highest as possible. The discharge speed near the intersection therefore should be increased as the shock wave $B C$ moves far from signal. This result suggests the further study regarding the need for improvement particularly for the case that detector is very close to signal. Even though the estimation results are not perfect, they provide some more closer between the estimated and the actual observed speed compared to not doing any adjustment as can be seen from Table 1 (column 2-5).

\section{Using Detector Data for Inverse Conversion from Observed Speed to Uninterrupted Speed}

The objective in this part is to convert the speed observed from detector $\left(v_{O}\right)$ so that it behaves like the one when signal does not exist (i.e., uninterrupted speed, $v_{A}$ ). As discussed earlier, $v_{A}$ is randomly assigned as the input for model. The value of $F$ is treated in the same way as the above part.

The range of feasible region plays a significant role to the accuracy of model. At first, the conversion was performed without imposing any constraint. The result of this is shown in Figure 5. From this figure, if the linear and parabolic shock wave were performed without imposing constraint, the results are mostly worse than the original model as also shown in Table 1. The possible way to deal with this problem is to introduce the upper and lower constraints. The upper constraint was set as the free flow speed and the lower constraint was set to either the speed at saturation flow rate or the observed speed, depends on which one is higher. This modification thus improves the estimation capability of all models as can be seen in Figure 6 and in Table 1 . However, at $q_{A} / c$ $=0.6$ and 0.8 , the original model was still superior to the linear and parabolic model. 


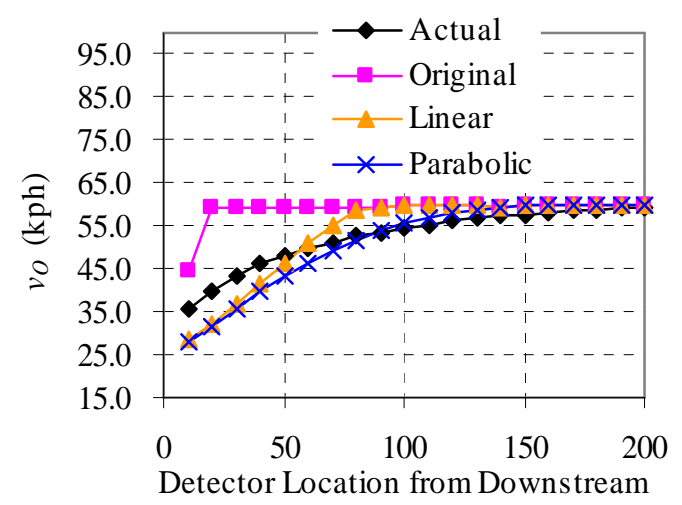

Signal (m)

(a) $q_{A} / c=0.2$

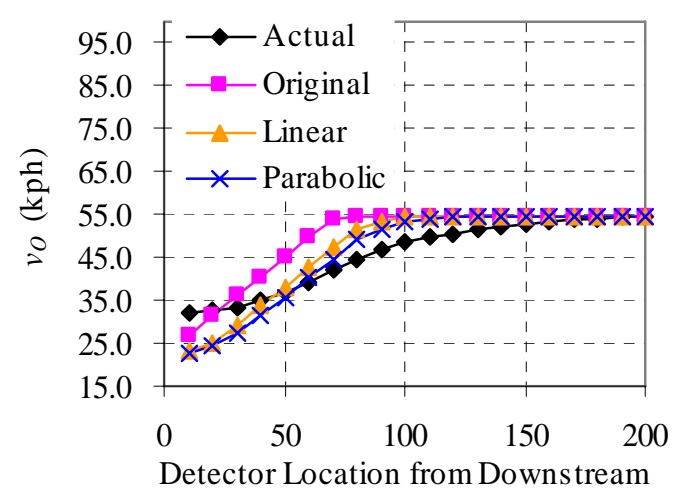

Signal (m)

(c) $q_{A} / c=0.6$

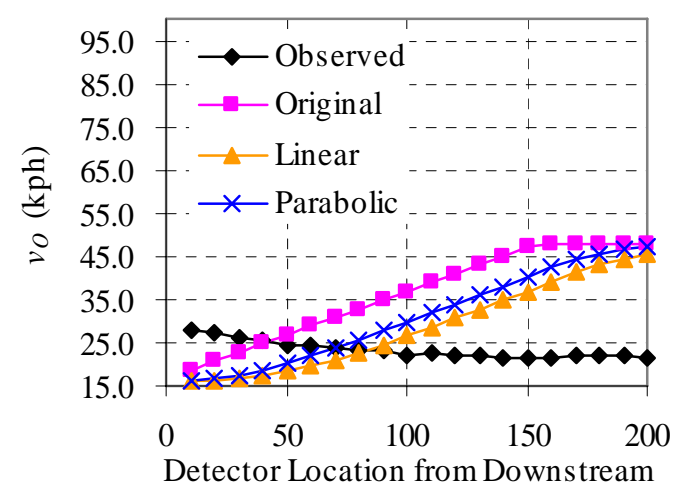

Signal (m)

(e) $q_{A} / c=1.0$

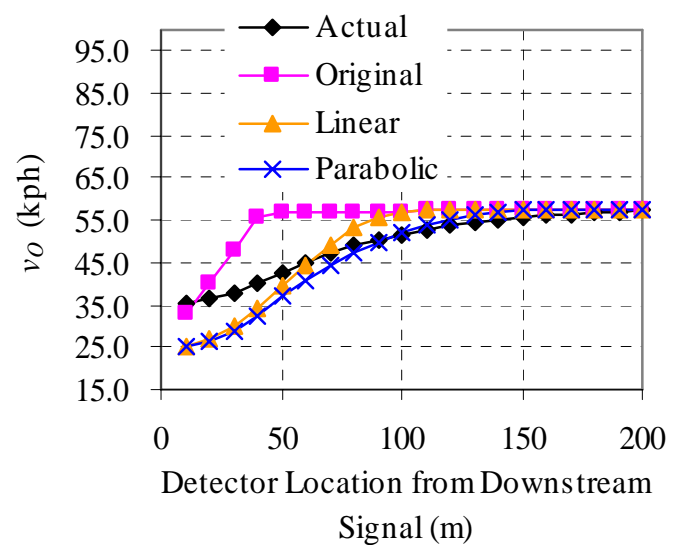

(b) $q_{A} / c=0.4$

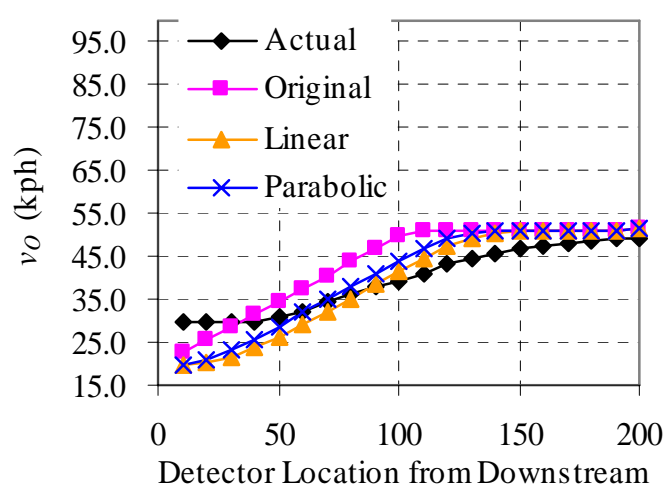

Signal (m)

(d) $q_{A} / c=0.8$

FIGURE 4 Using Detector Data for Direct Conversion from Uninterrupted Speed to Observed Speed. 


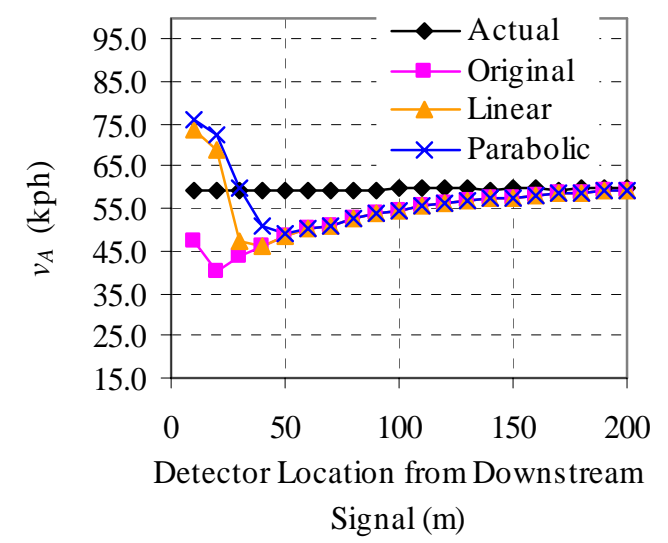

(a) $q_{A} / c=0.2$

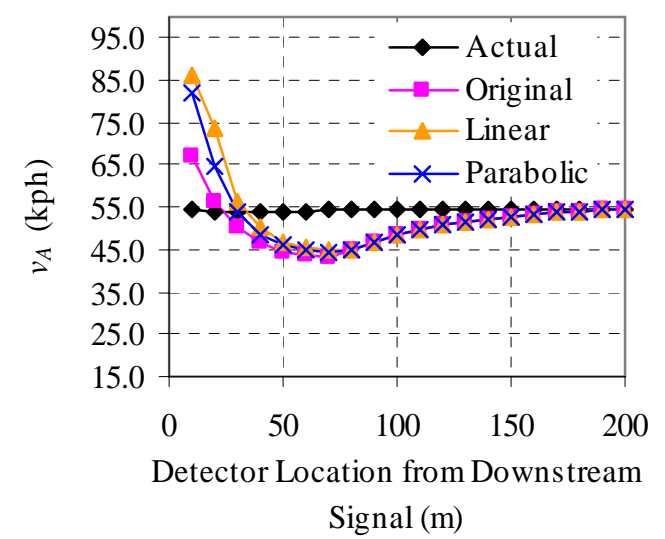

(c) $q_{A} / c=0.6$

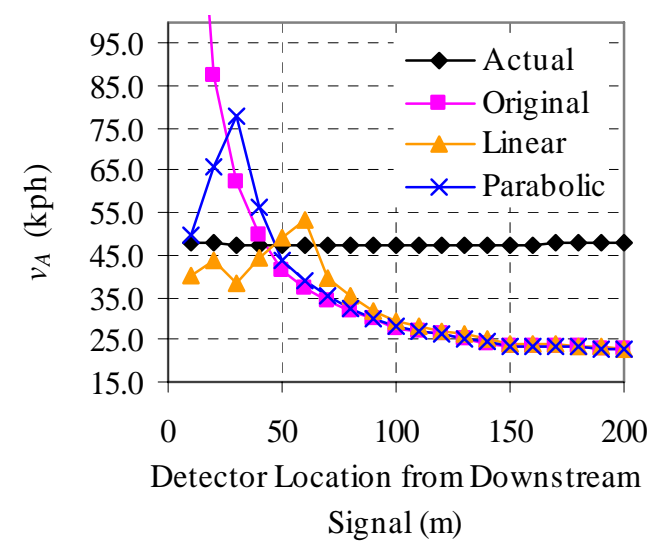

(e) $q_{A} / c=1.0$

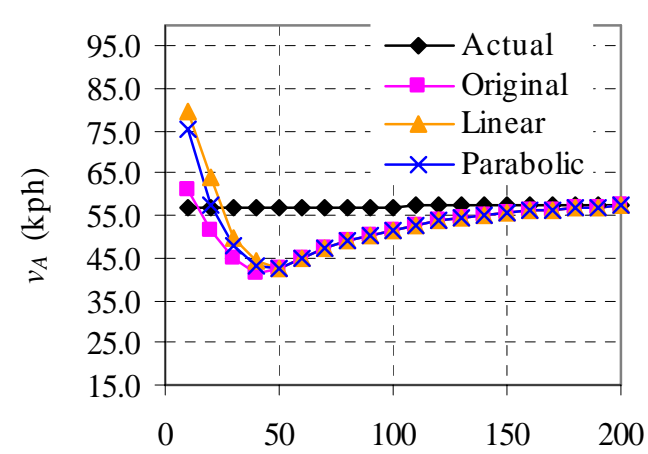

Detector Location from Downstream Signal (m)

(b) $q_{A} / c=0.4$

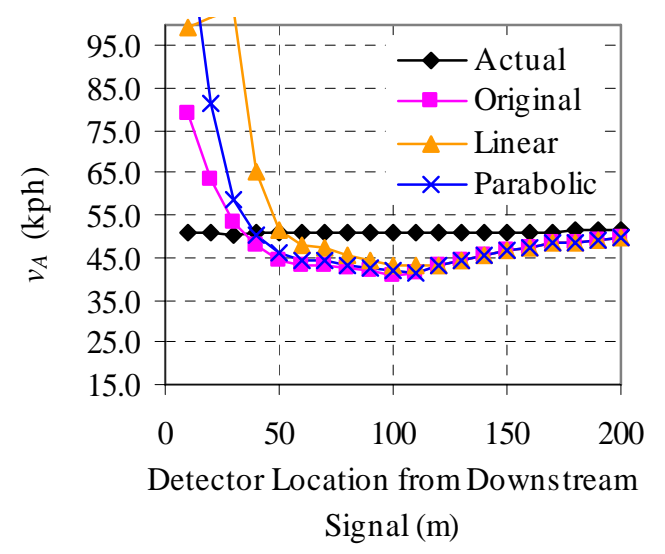

(d) $q_{A} / c=0.8$

FIGURE 5 Using Detector Data for Inverse Conversion from Observed Speed to Uninterrupted Speed (No Constraint). 


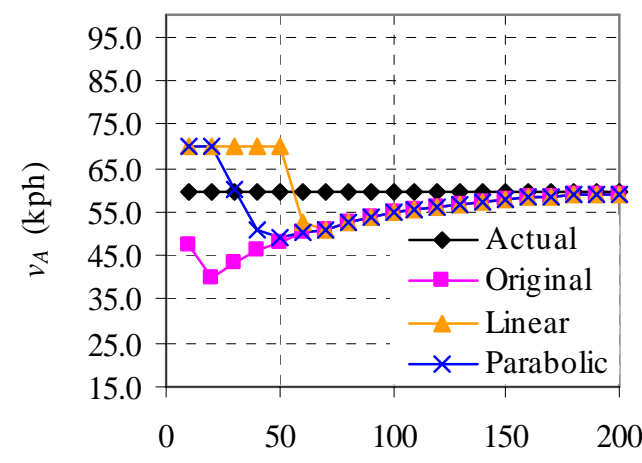

Detector Location from Downstream Signal (m)

(a) $q_{A} / c=0.2$

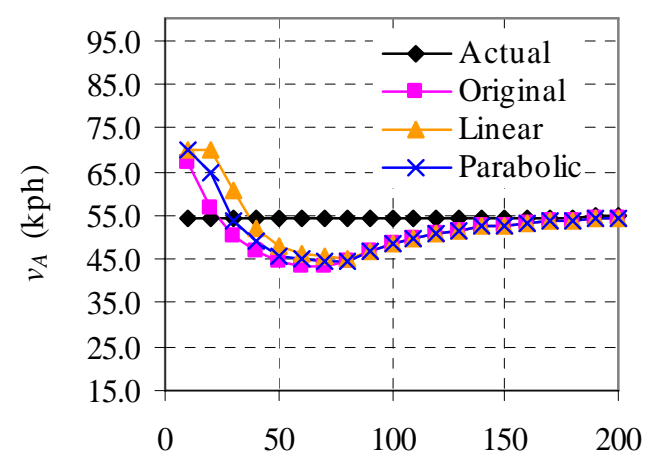

Detector Location from Downstream Signal (m)

(c) $q_{A} / c=0.6$

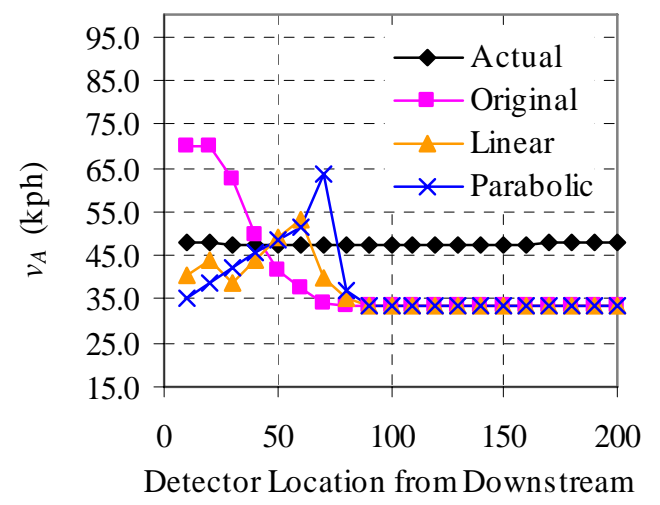

Signal (m)

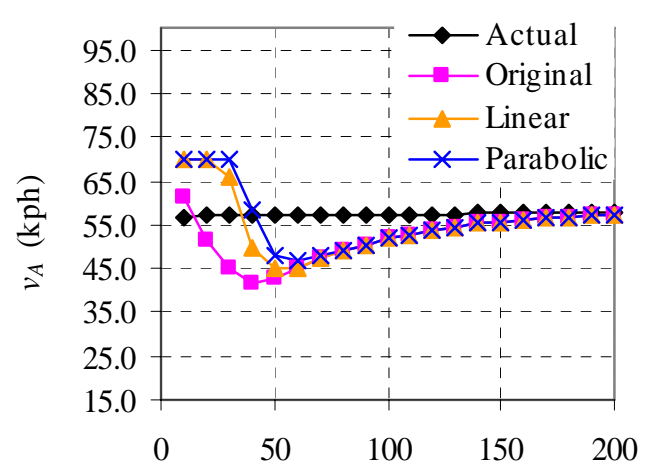

Detector Location from Downstream Signal (m)

(b) $q_{A} / c=0.4$

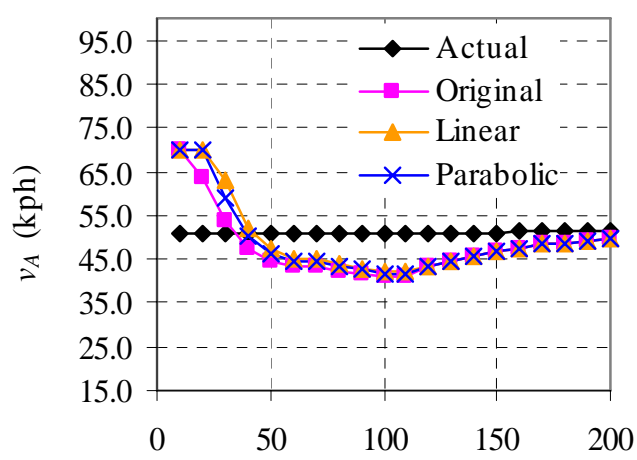

Detector Location from Downstream Signal (m)

(d) $q_{\mathrm{A}} / \mathrm{c}=0.8$

(e) $q_{A} / c=1.0$

FIGURE 6 Using Detector Data for Inverse Conversion from Observed Speed to Uninterrupted Speed (With Constraints) 
It also can be seen that the estimation results were not perfect for the case that detector was located near to signal. This result is similar to the direct conversion part and the same reason can be applied. All three models provide some improvements compared to the case of directly using observed speed as the uninterrupted speed (no adjustment). However, when comparing the results of this part and the direct conversion part, it can be seen that the direct conversion method provides better results than those obtained from this part, except only the case $q_{A} / c$ $=1.0$.

\section{Combination of Probes and Detector Data for Conversion from Observed Speed to Uninterrupted Speed}

As can be seen from the previous part, the use of only detector data to estimate the uninterrupted speed still needs some improvements particularly for the case that detector is installed near to signal. The use of probe data in addition to detector data is proposed in this paper with the hope to increase accuracy of model. Arrival flow rate obtained from detector was used to calculate average delay using equation (10) and the average observed speed from probes was then transformed to $v_{A}$ using equation (11). It can be seen from Table 1 (last column) that the use of two data sources has great significance on the improvement of the conversion from observed speed to uninterrupted speed. The percentage of probes used in this study was 5 percent. In real field, however, the percentage of probes is considerably lower than this number and thus limits the application of this method. If the percentage of probes is sufficiently high, the use of this method is preferable.

\section{Possible Applications of the Proposed Methods}

One of the possible applications of the proposed methods is the estimation of uninterrupted traffic state using the observed interrupted traffic data on urban road. The relationship between flow and speed, as the example of this application, was developed for different detector locations (50, 100, and $200 \mathrm{~m}$ from signal) as shown in Figure 7. The actual relationship obtained from uninterrupted traffic state is shown in Figure 7(a) and the plot of flowspeed for the case of no adjustment and parabolic model (with constraints) are shown in Figure 7(b), and 7(c), respectively. It can be seen that if traffic data from interrupted traffic state was plotted directly, the result is quite far from the actual diagram. After conversion, the result becomes closer to actual diagram but still need some improvements. As expected, the result of $q-v$ curve at $200 \mathrm{~m}$ is generally better than the result at the distance closer to signal. This results from the fact that the farther distance from stop line has less interference from congestion. However, the result at $50 \mathrm{~m}$ at the flow rate 800 and $1000 \mathrm{vph}$ appeared better than at the other distances. This happens because the converted speed changes from overestimated to underestimated at around this distance (see Figure 6). Further investigation is required to identify the main cause of such behavior.

The other application is the link travel time estimation. By knowing detector location, arrival flow rate, and average observed speed from detector, it is possible to estimate the arrival speed using the proposed method. Then cruise travel time can be obtained from the link length divided by arrival speed. Finally, calculating link delay due to signal (by shock wave concept or other delay formulas, see (9) for reviews of these formulas) and added to the cruise time, the average link travel time can then be obtained. Link travel time estimation using parabolic model with constraints and delay from shock wave was compared with the one using Zhang (2)'s model. To do this, another set of traffic data was simulated and used for calibration of parameters in Zhang's model. The estimated value of parameters $u_{f}, \alpha$, and $\beta$ in Zhang(2) are as $70 \mathrm{kph}, 12.94 \mathrm{kph}$, and 1.03, respectively. The actual link travel time was obtained by calculating the average travel time from all vehicles (all vehicles were flagged as probes). The results of actual link travel time and the estimated travel time from detector data at 50 and $200 \mathrm{~m}$ from both models are shown in Figure 8. From this figure, it can be seen that the proposed model outperforms Zhang's model in almost cases except at $q_{A} / c=1.0$. Though the accuracy of speed transformation is not sufficiently high, the results in this example suggest the potential of applying the proposed method for link travel time estimation. 


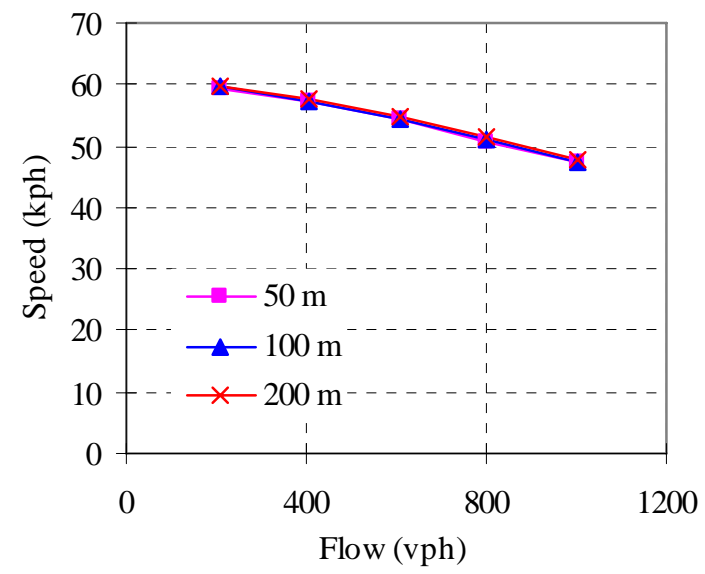

(a) Uninterrupted Flow

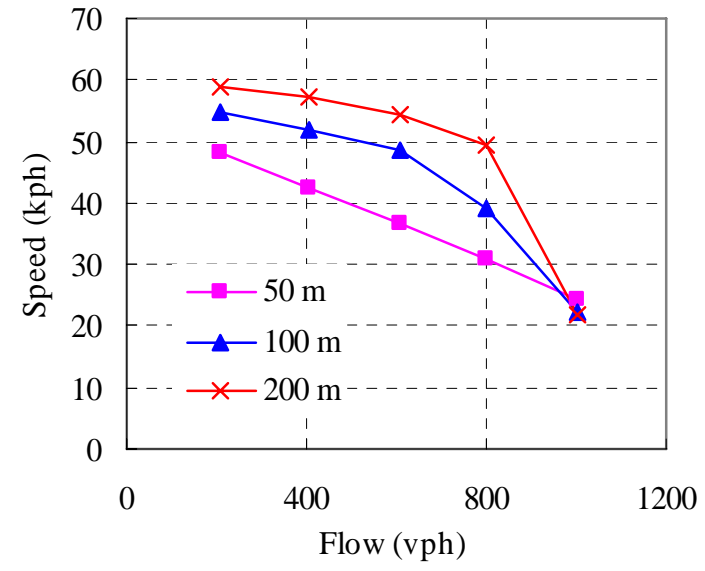

(b) Interrupted Flow (No Adjustment)

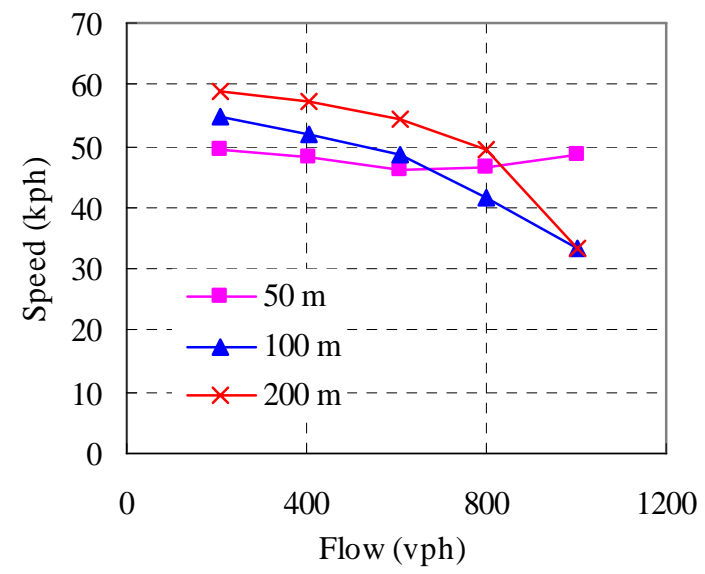

(c) Parabolic Model

FIGURE 7 Application of the Proposed Method for Speed-Flow Diagram. 


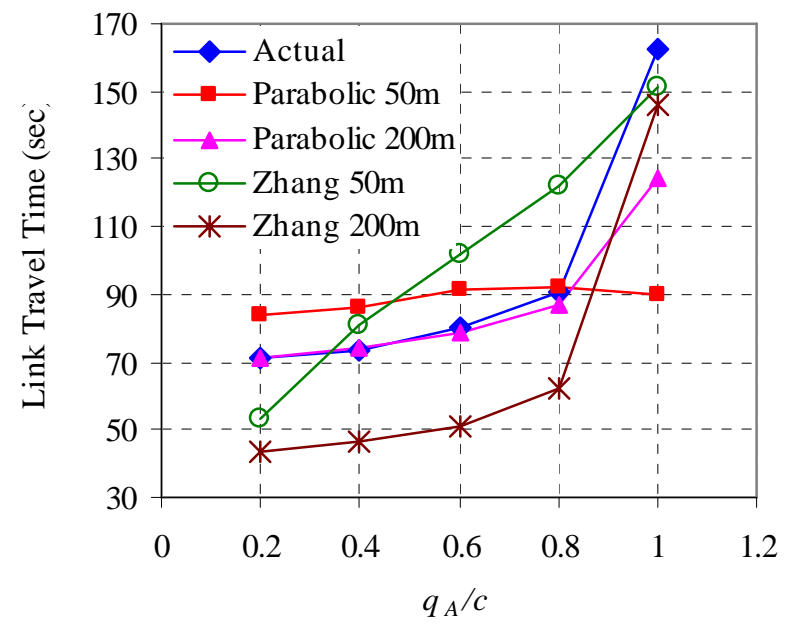

FIGURE 8 Application of the Proposed Method for Link Travel Time Estimation.

\section{CONCLUDING REMARKS}

Motivated from the lack of methodology to fully utilize available traffic data obtained from loop detector on urban roads, this paper attempted to transform between the uninterrupted and interrupted speed as well as to illustrate its potential applications. This paper, as a first stage, considered only the case of under-saturated traffic condition. The proposed method used shock wave theory as a fundamental background to relate the uninterrupted speed to interrupted speed. Conventional boundary and two modified boundaries of shock wave, linear and parabolic, were considered in the model development. An alternative way of integrating probes data with detector data was also discussed. The example applications of the proposed method on flow-speed diagram development and link travel time estimation were illustrated. Major findings are summarized here.

- $\quad$ The average observed speed tends to increase as the observation location is far away from downstream signal. However, at high demand, it exhibits the decreasing pattern at the distance near to signal and increasing pattern at the far distance.

- The results of speed transformation were in fairly good agreement at the far observation location. However, large difference can still be seen at the observation point near to signal.

- If both probes and detector data were available, the method of integrating both data sources was most effective.

- If only detector data was available, direct conversion from uninterrupted speed to the average observed speed was more accurate.

- The proposed methods have a potential of being applied for link travel time estimation.

Even though the results from numerical suggested some agreements, there were still some differences particularly when detector location was near to signal. The problems leading to this result as well as the limitations of this method are noted here:

- The assumption of instantaneously changing speed from stop to a constant speed at saturation flow rate is not realistic. This problem was expected to be the major factor of low accuracy at the observation location near to signal.

- $\quad$ This method is applicable only for the case of under-saturated condition.

- It is assumed that there is no obstruction from opposing right-turn vehicles. Therefore, traffic stream is interrupted only by signal.

- All models were developed based on the case of isolated signalized intersection with long straight approaching links and constant arriving demand during the aggregated interval.

In order to make the model more realistic, the arrival demand pattern should be adjusted in the way that corresponds to the discharge rate and effective green interval of the upstream signal and also the time offset between upstream and downstream signals. This issue can be performed by considering the case of successive 
signalized intersections with and without signal time offset. Extensive numerical experiments by varying signal settings, link characteristics, and demand patterns should be conducted with specific focus on the section near to signal. Shock wave method based on characteristic wave can also be considered as one candidate to overcome the first limitation listed above. Another way is to consider the optimal detector location that would provide sufficiently high accuracy. All the above mentions as well as the verification of the proposed method using real traffic data are suggested for future research.

\section{REFERENCES}

1. May, A. D. Traffic Flow Fundamentals. Prentice-Hall, Inc., Englewood Cliffs, New Jersey, 1990.

2. Zhang, H. M. Link-Journey-Speed Model for Arterial Traffic, Transportation Research Record 1676, 1999, pp. 109-115.

3. Sisiopiku, V. P., Rouphail, N. M., and Santiago, A. Analysis of Correlation Between Arterial Travel Time and Detector Data from Simulation and Field Studies, Transportation Research Record 1457, 1994, pp. 166-173.

4. Xie, C., Cheu, R. L., and Lee, D-H. Calibration-Free Arterial Link Speed Estimation Model Using Loop Data, Journal of Transportation Engineering, Vol. 127, No. 6, 2001, pp. 507-514.

5. Lighthill, M. H. and Whitham, G. B. On Kinematic Waves-II. A Theory of Traffic Flow on Long Crowded Roads, Proceedings of the Royal Society, Series A, Vol. 229, 1957, pp. 317-345. (Reprinted in Highway Research Board, Special Report 79, HRB, Washington, D.C., 1964, pp. 7-35.

6. Richards, P. I. Shock Waves on the Highway, Operations Research, Vol. 4, No. 1, 1956, pp. 42-51.

7. Stephanopoulos, G. and Michalopoulos, P. G. Modelling and Analysis of Traffic Queue Dynamics at Signalized Intersections, Transportation Research, Vol. 13A, 1979, pp. 295-307.

8. INTEGRATION Release 2.30 User’s Guide I \& II: Fundamental Model Features \& Advanced Model Features. M.Van Aerde \& Assoc., Ltd., May, 2001.

9. Dion, F., Rakha, H., and Kang, Y-S. Comparison of Delay Estimates At Under-Saturated and OverSaturated Pre-Timed Signalized Intersections, Transportation Research Part B, Vol. 38, No. 2, 2004, pp. 99122. 